\title{
Clinical Application of Microsurgery Using the Cerebellar Medulla Fissure Approach in Severe Ventricular Hemorrhage with Casting of the Fourth Ventricle and Its Influence on Neurological Recovery
}

\author{
Feng Gao, Huixiao Wang, and Zhengzheng Wang \\ Department of Neurosurgery, The Affiliated People's Hospital of Ningbo University, Ningbo, Zhejiang 315040, China \\ Correspondence should be addressed to Zhengzheng Wang; wangzhengzheng53@sohu.com
}

Received 8 September 2021; Accepted 30 September 2021; Published 25 October 2021

Academic Editor: Songwen Tan

Copyright $\odot 2021$ Feng Gao et al. This is an open access article distributed under the Creative Commons Attribution License, which permits unrestricted use, distribution, and reproduction in any medium, provided the original work is properly cited.

\begin{abstract}
Objective. To investigate the clinical application of microsurgery using the cerebellar medulla fissure approach in severe ventricular hemorrhage with casting of the fourth ventricle and its effect on neurological recovery. Methods. A total of 80 patients with severe intraventricular hemorrhage accompanied by casting and dilation of the fourth ventricle who were admitted to the neurosurgery department between July 2019 and December 2020 were randomly divided into an observation group and a control group, with 40 patients in each group. The drainage tube extubation time and length of hospital stay of the two groups were compared. The 3-day hematoma clearance of the two groups was observed. Postoperative consciousness was evaluated by GCS, and the patients' recovery was evaluated by GOS at three months. The activities of daily living (ADL) scores of the two groups were compared to evaluate patients' postoperative self-care ability. The incidence of postoperative complications in the two groups was collected. Independent risk factors for poor prognosis were analyzed by logistics regression. Results. The postoperative hospitalization time and the drainage tube extubation time in the observation group were significantly lower than those in the control group. The ratio of hematoma clearance $\geq 90 \%$ in the observation group was significantly higher than that of the control group. Postoperative GCS scores and GOS scores in the observation group were significantly higher than those of the control group. The rate of postoperative complications in the observation group was significantly lower than that of the control group. The rate of good ADL grading in the observation group was significantly higher than that in the control group. Age and surgical method were independent risk factors for poor prognosis. Conclusion. Microsurgery using the cerebellar medulla fissure approach can effectively improve the condition of severe ventricular hemorrhage with casting of the fourth ventricle and promote the recovery of patients' neurological function.
\end{abstract}

\section{Introduction}

Intracerebral hemorrhage is a relatively common but extremely dangerous disease in clinical practice. In many patients, spontaneous intracerebral hemorrhage is caused by nontraumatic factors, and their mortality and disability rates are extremely high [1-3]. If an intracerebral hemorrhage breaks into the ventricle, it may quickly lead to a rapid increase in intracranial pressure, acute dilation of the ventricle, and compression of the ventricle system by a hematoma, resulting in acute obstructive hydrocephalus
$[4,5]$. These patients will develop critical disease quickly, with rapid onset, rapid progression, high mortality, and poor prognosis [6].

In patients with ventricular hemorrhage, ventricular casts tend to form, especially in patients with blood clots in the third and fourth ventricles; these patients develop blockage of cerebrospinal fluid circulation and compression of the brainstem [7], leading to high mortality and disability rates with traditional treatment; therefore, it is necessary to find a more effective treatment to improve the prognosis of the disease. 
At present, the commonly used clinical treatment is a ventricular puncture and an injection of urokinase to dissolve and drain the hematoma or even lumbar cistern drainage in severe patients $[8,9]$. However, this treatment is not effective for patients with severe ventricular hemorrhage who develop a fourth ventricle cast. Traditional treatments take a long time to eliminate the hematoma, resulting in continued compression and irreversible damage to the brainstem [10, 11]. In recent years, some scholars have explored the removal of the hematoma of the fourth ventricle through cerebellar vermis incision, which has saved the lives of some critically ill patients, but this surgical approach may lead to cerebellar vermis injury [12]. On the other hand, in the cerebellar medulla fissure approach, there is no need to cut the vermis of the cerebellum, but the natural space of the cerebellar medulla fissure is separated to remove the hematoma of the fourth ventricle. Through the placement of the drainage tube in the cerebellar medulla system, brain stem compression can be reduced in the shortest possible time, and the blockage of cerebrospinal fluid circulation can be relieved [13].

Therefore, in this study, microsurgery was performed on patients with severe ventricular hemorrhage accompanied by casting of the fourth ventricle using the cerebellar medulla fissure approach to observe the short- and long-term efficacy of this approach.

\section{Method}

2.1. Clinical Data of Patients. A total of 80 patients with severe intraventricular hemorrhage accompanied by casting and dilation of the fourth ventricle who were admitted to the neurosurgery department between July 2019 and December 2020 were randomly divided into an observation and a control group, with 40 cases in each group. The control group consisted of 23 males and 17 females (mean age: $58.54 \pm 7.31$ ) years, while the observation group consisted of 25 males and 15 females (mean age: $58.19 \pm 8.73$ ) years. The control group was treated with traditional ventricular puncture and drainage, urokinase lysis, and lumbar cistern drainage. The observation group underwent ventricular puncture and drainage, urokinase lysis, and removal of hematoma in the fourth ventricle through cerebellomedullary fissure approach. The study followed the Declaration of Helsinki and was approved by the ethics committee of our hospital. Both patients and their families were informed about the study and provided their written consent to participate in the study.

2.2. Inclusion Criteria and Exclusion Criteria. Inclusion criteria were as follows: onset time $<24 \mathrm{~h}$; age $>18$; plain CT scan showing bleeding into the ventricle, with parenchymal bleeding $<30 \mathrm{ml}$ and a dilated hematoma in the fourth ventricle [14].

Exclusion criteria were as follows: patients with secondary cerebral hemorrhage due to arteriovenous malformation, ruptured intracranial aneurysm, craniocerebral injury, brain tumor, or hemorrhagic infarction; patients who did not meet the surgical indications; patients with anticoagulation-related cerebral hemorrhage; patients who refused to follow up for clinical evaluation after discharge; severe hepatic and renal insufficiency; pregnant or lactating women with other malignant tumors.

2.3. Treatment Options. The control group received traditional ventricle puncture drainage plus urokinase lysis plus lumbar cistern drainage. Observation group used urokinase dissolution ventricular puncture and drainage plus the fourth ventricle cerebellomedullary fissure drainage to remove the hematoma. In the prone position of the patient, an incision was made in the middle of the posterior occipital region, and the foramen magnum was opened (in the case of tonsillar hernia, the posterior arch of Atlas was opened). The dura mater was cut in a Y shape and suspended to the upper and both sides. The hematoma in the cerebellar parenchyma was removed under the microscope, and the structures such as bilateral cerebellar hemispheres, vermis, cerebellar tonsil, medulla oblongata, and posterior inferior cerebellar artery were exposed. Cerebrospinal fluid was released from the arachnoid membrane in the greater occipital pool under the microscope. The arachnoid membranes around the cerebellomedullary fissure and cerebellomedullary pool were sharply separated, and the bilateral vermis tonsil space and medullary tonsil space were separated, so that the mobility of cerebellar tonsil was increased. The choroid was cut along the center and the border of the membranous medullary sail up to the upper margin of the lower medullary sail. The choroidal zone was cut from the latch toward the lateral recess, or it could be expanded to the posterior edge of the lateral recess and the lateral margin of the lower medullary sail. The cerebellar tonsil and vermis were retracted and fixed sideward and upward, and the choroid and inferior medullary sail were cut open to fully expose the bottom of the fourth ventricle. During the operation, the mesencephalic aqueduct was explored, and the hematoma was suctioned out without contact. No. 8 ventricular drainage tube was placed in the mesencephalic aqueduct, and the ventricular system was rinsed and dredged with warm normal saline. During the operation, the bleeding was stopped completely, and the fourth ventricle drainage tube was retained. The dura mater was tightly sutured, layer by layer until reaching the scalp. The epidural drainage tube was also retained. Postoperative antibiotics were routinely used to prevent infection, and oxygen inhalation, hemostasis, dehydration, and antihypertensive treatment were given. Meanwhile, nasogastric feeding or intravenous nutrition support was given to maintain the water and electrolyte balance. After the operation, the control group and the observation group received the same drugs and other treatments, including routine ECG and intracranial pressure monitoring, oxygen inhalation, dehydration to reduce intracranial pressure, nutrition of brain cells, prevention and treatment of rebleeding, drugs to relieve cerebral vasospasm, and active prevention and treatment of various complications and symptoms. 
2.4. Observation Index. The drainage tube extubation time and length of hospital stay for the two groups were compared. Twenty-four hours after surgery, a head CT was performed to observe the 3-day hematoma clearance rate. The 3-day hematoma clearance rate $=$ (preoperative hematoma volume-postoperative hematoma volume)/preoperative hematoma volume $\times 100 \%$. Postoperative consciousness was evaluated by GCS score, and the patients' recovery was evaluated by GOS score at three months [15]. Patients' self-care ability was evaluated with an ADL score, where I + II + III = a "good" prognosis; other scores indicated a poor prognosis [16]. All patients were followed up for 3 months after operation, and the incidence of postoperative complications such as hydrocephalus, pulmonary infection, intracranial infection, and gastrointestinal bleeding in both groups was recorded.

2.5. Statistical Method. The results of this experiment were statistically analyzed by SPSS 20.0 (SPSS Co., Ltd., Chicago, USA). Count data were expressed by (rate), and chi-square test was used for their comparison between groups. Measurement data were expressed by (mean \pm standard deviation), and $t$-test was used for their comparison between groups. Multivariate analysis adopts multiple Logistic regression model. $P<0.05$ indicates that the difference is statistically significant.

\section{Results}

3.1. Patient Baseline Datasheet. A comparison of the clinical data of the two groups revealed no statistical difference between the two groups in terms of gender, age, time of onset, bleeding site, grab score, previous medical history, GCS score, coma, and intraventricular hemorrhage, as shown in Table 1.

3.2. Comparison of Surgical Indications. A comparison of the operation indications of the two groups revealed that the drainage tube extubation time and length of hospital stay of the observation group were significantly lower than those of the control group, and the difference is statistically significant, as shown in Figure 1.

3.3. Comparison of 3-Day Hematoma Clearance Rate in Each Group. A comparison of the 3-day hematoma clearance rate of the two groups revealed that the rate of patients with a 3 day hematoma clearance rate $\geq$ of $90 \%$ in the observation group was significantly higher than that in the control group, and the difference is statistically significant, as shown in Table 2.

3.4. Comparison of Postoperative Consciousness and Recovery of Patients. The postoperative consciousness of the two groups was evaluated by postoperative GCS scores. It was found that the postoperative GCS scores of the observation group were significantly higher than those of the control group. The postoperative recovery of the two groups was evaluated by the GOS score after three months. It was found that the GOS score of the observation group was significantly higher than that of the control group, and the difference is statistically significant, as shown in Figure 2.

3.5. Comparison of the ADL Scores of Patients. The postoperative self-care ability of the patients was evaluated by ADL score. It was found that the rate of a "good" classification for patients in the observation group was significantly higher than that of the control group. The difference was statistically significant, as shown in Table 3.

3.6. Comparison of Postoperative Complications. A comparison of the postoperative complications of the two groups, including hydrocephalus, pulmonary infection, intracranial infection, and gastrointestinal bleeding, revealed that the incidence of complications in the observation group was significantly lower than that in the control group, as shown in Table 4.

3.7. Analysis of Risk Factors for Poor Prognosis. The patients were divided into a 'good prognosis group' and 'poor prognosis group' according to their ADL score. The clinical data of the two groups were included in the univariate analysis (Table 5). Statistical differences were found between the two groups in terms of age, admission GCS score, amount of intraventricular hemorrhage, and amount of hemorrhage. When the factors with statistical differences were included in the logistic regression equation for multivariate analysis, age and operation mode were found to be independent risk factors for poor prognosis, as shown in Tables 6 and 7 .

\section{Discussion}

The development of ventricular casting caused by ventricular hemorrhage is very fast, the prognosis of patients is often poor, and the disability rate is very high [17]. Therefore, the treatment plan is often to relieve the compression of the brainstem, dredge the obstruction of the ventricle, reduce the patient's brain edema, and reduce intracranial pressure [18]. The traditional treatment scheme results in improvement for some patients, but the disability rate of patients is still high. In patients with casting of the fourth ventricle, intraventricular hematocele will compress the brainstem and affect the perfusion of the perforator artery of the brainstem, resulting in insufficient blood supply to the brainstem and ischemic necrosis [19].

The lumbar cistern puncture and drainage plus urokinase lysis treatment used in the past can reduce the compression on the brainstem, reduce brain edema, and release intracranial hypertension to a certain extent, but it takes one to two days to remove the hematoma in the fourth ventricle, which will result in serious damage to the patient's brainstem [20]. At the same time, urokinase can accelerate the dissolution of clots, but it is easy to increase the risk of rebleeding and intracranial infection [21]. Fountas et al. [22] 
TABle 1: Patient baseline datasheet.

\begin{tabular}{|c|c|c|c|c|}
\hline & Control group $(n=40)$ & Observation group $(n=40)$ & $X^{2} / t$ & $P$ \\
\hline Gender & & & 0.208 & 0.648 \\
\hline Male & $23(57.50)$ & $25(62.50)$ & & \\
\hline Female & $17(42.50)$ & $15(37.50)$ & & \\
\hline Age (years) & $58.54 \pm 7.31$ & $58.19 \pm 8.73$ & 0.194 & 0.846 \\
\hline Time of onset (h) & $6.14 \pm 2.20$ & $6.42 \pm 2.23$ & 0.565 & 0.574 \\
\hline Bleeding & & & 0.287 & 0.592 \\
\hline The cerebellum & $10(25.00)$ & $8(20.00)$ & & \\
\hline The thalamus & $30(75.00)$ & $32(80.00)$ & & \\
\hline Great score & $6.23 \pm 1.22$ & $6.18 \pm 1.05$ & 0.196 & 0.845 \\
\hline Medical history & & & 0.474 & 0.789 \\
\hline Hypertension & $6(15.00)$ & $7(17.50)$ & & \\
\hline Diabetes & $8(20.00)$ & $6(15.00)$ & & \\
\hline Hyperlipidemia & $4(10.00)$ & $5(12.50)$ & & \\
\hline GCS score on admission & $8.51 \pm 2.65$ & $8.56 \pm 3.39$ & 0.073 & 0.942 \\
\hline Admitted to hospital in a coma & & & 0.503 & 0.478 \\
\hline Yes & $25(62.50)$ & $28(70.00)$ & & \\
\hline No & $15(37.50)$ & $12(30.00)$ & & \\
\hline Ventricular hemorrhage $(\mathrm{mL})$ & $18.03 \pm 5.11$ & $18.27 \pm 4.58$ & 0.221 & 0.826 \\
\hline
\end{tabular}

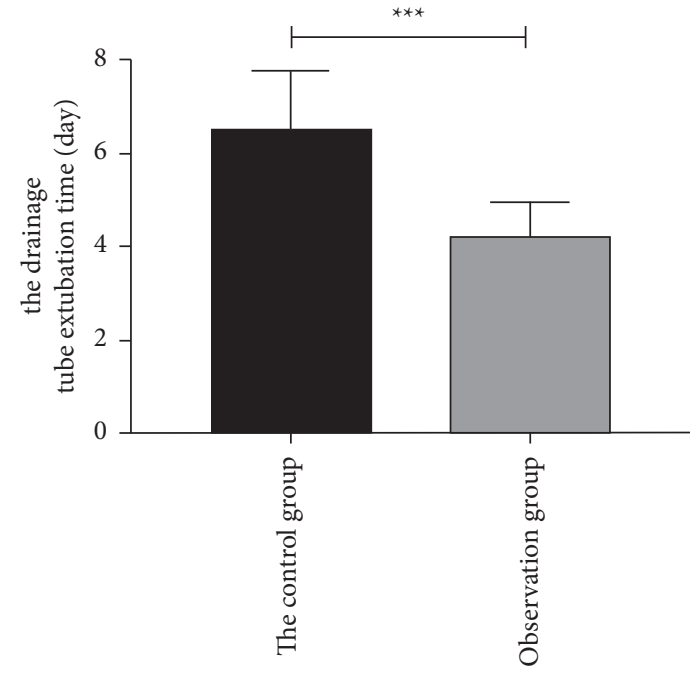

(a)

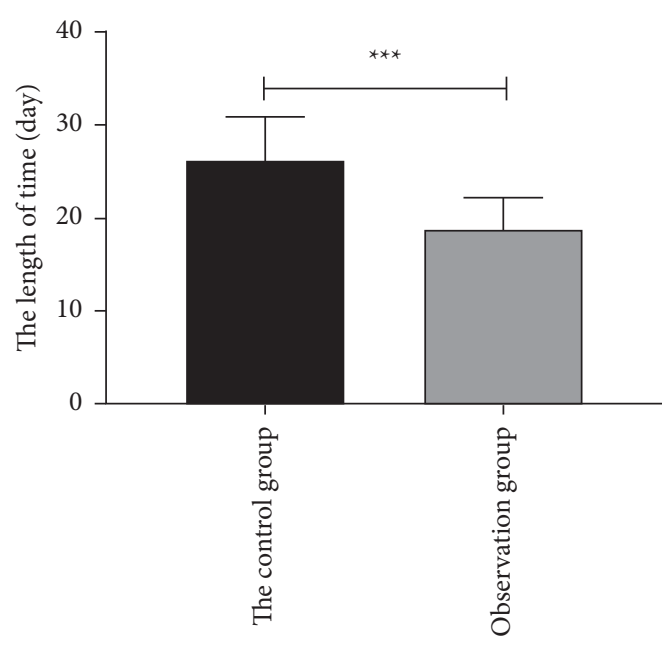

(b)

Figure 1: Comparison of surgical indications. (a) The drainage tube extubation time in the observation group was significantly lower than that in the control group $(P<0.001)$. (b) The length of stay in the observation group was significantly lower than that in the control group $(P<0.001)$. Note. ${ }^{* * *} P<0.001$.

TABLE 2: Comparison of the 3-day hematoma clearance rate in each group.

\begin{tabular}{lccr}
\hline 3-day hematoma clearance rate & Control group $(n=40)$ & Observation group $(n=40)$ & $X^{2}$ \\
\hline$\geq 90 \%$ & $21(52.50)$ & $30(75.00)$ & 4.381 \\
$<90 \%$ and $\geq 70 \%$ & $13(32.50)$ & $8(20.00)$ & 0.036 \\
$<70 \%$ & $6(15.00)$ & $2(5.00)$ & 0.204 \\
\hline
\end{tabular}

observed that the use of urokinase brings a risk of bleeding and intracranial infection. Compared with microsurgery, conventional surgery is more traumatic, and intraoperative injury often leads to respiratory, circulatory, and consciousness dysfunction. In addition, in the traditional treatment, the cerebellar vermis needs to be cut, which may lead to complications such as cerebellar mutism or ataxia. The incidence of other postoperative complications is high, which is not conducive to the recovery of patients. Even when patients' lives can be saved, these complications may lead to lifelong disability [23]. With the development of medical technology, microsurgery has been applied to 


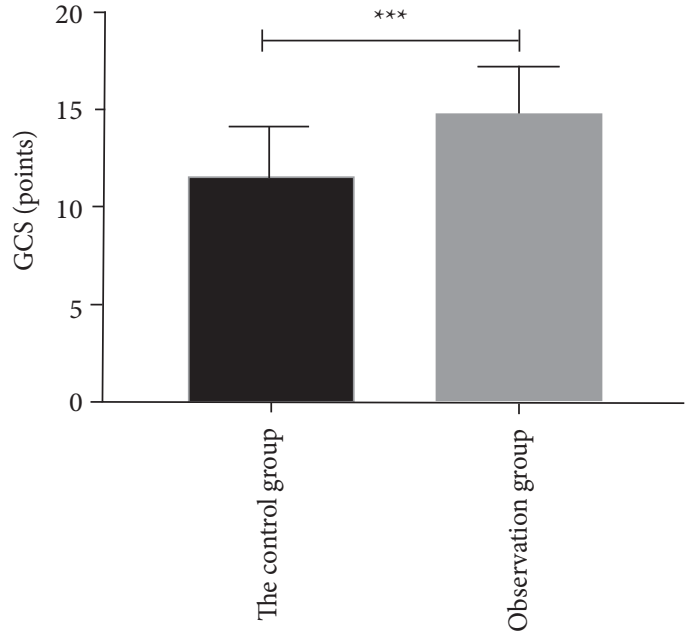

(a)

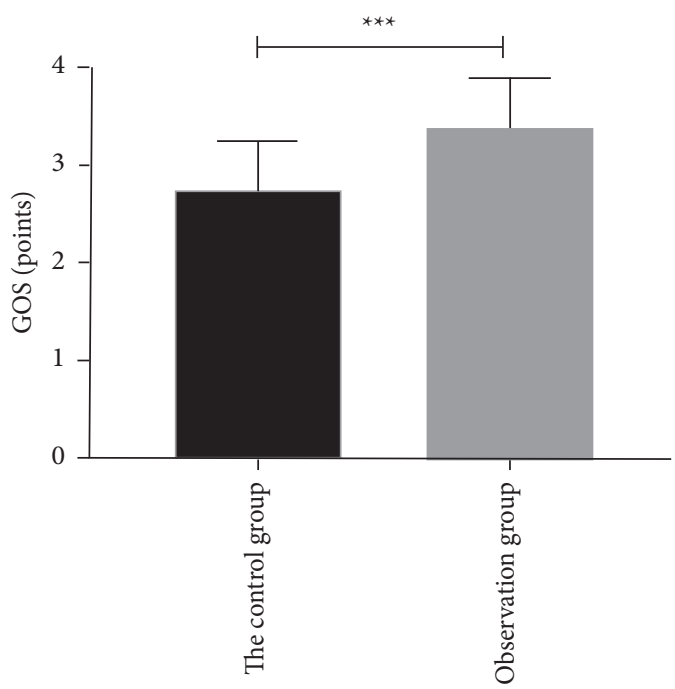

(b)

FIGURE 2: Comparison of postoperative consciousness and recovery of patients. (a) The postoperative GCS score of the observation group was significantly higher than that of the control group $(P<0.001)$. (b) The GOS score of the observation group was significantly higher than that of the control group after 3 months $(P<0.001)$. Note. ${ }^{* * *} P<0.001$.

TABle 3: Comparison of the ADL scores of patients.

\begin{tabular}{|c|c|c|c|c|c|c|}
\hline & $\mathrm{I}$ & II & III & IV & $\mathrm{V}$ & Good prognosis \\
\hline Control group $(n=40)$ & $3(7.50)$ & $6(15.00)$ & $8(20.00)$ & $16(40.00)$ & $7(17.5)$ & $17(42.50)$ \\
\hline Observation group $(n=40)$ & $6(15.00)$ & $8(20.00)$ & $13(32.50)$ & $10(25.00)$ & $3(7.50)$ & $27(67.50)$ \\
\hline$X^{2}$ & & & & & & 5.501 \\
\hline$P$ & & & & & & 0.025 \\
\hline
\end{tabular}

TABLE 4: Comparison of postoperative complications.

\begin{tabular}{lccc}
\hline & Control group $(n=40)$ & Observation group $(n=40)$ & $X^{2}$ \\
\hline Hydrocephalus & $4(10.00)$ & $1(2.50)$ & $P$ \\
Lung infection & $5(12.50)$ & $2(5.00)$ & $0(0.00)$ \\
Intracranial infection & $3(7.50)$ & $2(5.00)$ & $5(12.50)$ \\
Gastrointestinal hemorrhage & $4(10.00)$ & $16(40.00)$ & 7.813 \\
Total complications & 16.005 \\
\hline
\end{tabular}

TABle 5: Single-factor analysis table.

\begin{tabular}{|c|c|c|c|c|}
\hline & $\begin{array}{l}\text { Poor prognosis group } \\
\qquad(n=31)\end{array}$ & $\begin{array}{c}\text { Good prognosis group } \\
(n=49)\end{array}$ & $X^{2} / t$ & $P$ \\
\hline Gender & & & 0.694 & 0.405 \\
\hline Male & $20(64.52)$ & $27(55.10)$ & & \\
\hline Female & $11(35.48)$ & $22(44.90)$ & & \\
\hline Age (years) & $60.71 \pm 6.70$ & $56.83 \pm 8.42$ & 2.167 & 0.033 \\
\hline Time of onset (h) & $6.51 \pm 2.02$ & $5.80 \pm 2.33$ & 1.396 & 0.167 \\
\hline Bleeding & & & 2.794 & 0.097 \\
\hline The cerebellum & $10(32.26)$ & $8(16.33)$ & & \\
\hline The thalamus & $21(67.74)$ & $41(83.67)$ & & \\
\hline Great score & $6.22 \pm 1.99$ & $6.20 \pm 1.10$ & 0.058 & 0.954 \\
\hline Medical history & & & 0.474 & 0.789 \\
\hline Hypertension & $6(19.35)$ & 7 (14.29) & & \\
\hline Diabetes & $8(25.81)$ & $6(12.24)$ & & \\
\hline Hyperlipidemia & $4(12.90)$ & $5(10.20)$ & & \\
\hline
\end{tabular}


TABLE 5: Continued.

\begin{tabular}{lccc}
\hline & $\begin{array}{c}\text { Poor prognosis group } \\
(n=31)\end{array}$ & $\begin{array}{c}\text { Good prognosis group } \\
(n=49)\end{array}$ & $X^{2} / t$ \\
\hline GCS score on admission & $7.35 \pm 2.65$ & $9.28 \pm 3.03$ & 2.910 \\
Admitted to hospital in a coma & & 0.005 \\
$\quad$ Yes & $21(67.74)$ & $32(65.31)$ & \\
$\quad$ No & $10(32.26)$ & $17(34.69)$ & 0.050 \\
0.822 \\
Ventricular hemorrhage (mL) & $21.10 \pm 4.70$ & $16.28 \pm 3.90$ & $4.970<0.001$ \\
Operation mode & & $17(34.69)$ & $11.850<0.001$ \\
$\quad \begin{array}{l}\text { Traditional surgical methods } \\
\text { The microscopical approach of cerebellomedullary } \\
\text { fissure }\end{array}$ & $23(74.19)$ & $32(65.31)$ & \\
\hline
\end{tabular}

TABle 6: Assignment for multivariate analysis of factors.

\begin{tabular}{lcc}
\hline Factors & Variables & Assignment \\
\hline Age & $X 1$ & Continuous variable \\
Operation mode & $X 2$ & The microscopical approach of cerebellomedullary fissure $=0$ and traditional surgical methods $=1$ \\
\hline
\end{tabular}

TABLE 7: Multifactor analysis table.

\begin{tabular}{lcccccc}
\hline & $\mathrm{B}$ & $\mathrm{SE}$ & Wals & OR & $P$ & 95\%CI \\
\hline $\begin{array}{l}\text { Age } \\
\begin{array}{l}\text { Operation } \\
\text { mode }\end{array}\end{array}$ & 0.081 & 0.037 & 4.883 & 1.084 & 0.027 & $1.009 \sim 1.165$ \\
\hline
\end{tabular}

various cardiovascular and cerebrovascular diseases. In this study, the incidence of postoperative complications in the observation group was significantly lower than that in the control group, and the amount of intraoperative bleeding and the length of hospital stay were both significantly lower than those in patients who received the traditional treatment.

The cerebellomedullary fissure is a natural physiological fissure in the brain that is located before the cerebellar vermis node and cerebellar vermis sag. This microsurgery approach does not require that the lower cerebellar vermis be cut to obtain access to the operation field and to open the fourth ventricular floor. Thus, this approach can be used to remove a hematoma without damaging normal brain tissue [24]. The hematoma rate of the observation group was significantly better than that of the control group, indicating that the cerebellomedullary fissure approach can completely remove the hematoma in the cerebellum and the fourth ventricle to dredge the ventricular system and protect the brainstem function. The GCS scores and GOS scores of the observation group were higher than those of the control group after three months. The postoperative recovery of the patients was evaluated through their ADL scores. The rate of "good" scores in the observation group was significantly higher than that in the control group, indicating that the microsurgical treatment of the cerebellar medullary fissure approach is more conducive to a good prognosis. We also found that age and operation mode were the risk factors for an adverse prognosis.

This study has some limitations. First, the cerebellomedullary fissure microsurgery approach requires familiarity with the anatomical characteristics around the fourth ventricle. Second, a variety of neuroelectrophysiological monitoring needs to be combined during the operation to monitor the blood supply and brainstem function of patients. Therefore, the operation site needs to be adjusted [25].

In conclusion, the cerebellomedullary fissure microsurgery approach can effectively improve the condition of severe intraventricular hemorrhage with casting of the fourth ventricle and promote the recovery of neurological function.

\section{Data Availability}

The data used during the current study are available from the corresponding author upon request.

\section{Ethical Approval}

This study was approved by the ethics committee of The Affiliated People's Hospital of Ningbo University (2019016).

\section{Conflicts of Interest}

The authors declare no conflicts of interest.

\section{Acknowledgments}

This study was supported by the Ningbo Yinzhou District Agricultural and Social Development Science and Technology Project, under Grant no. 20191YZQ010003.

\section{References}

[1] Y. Cenik, A. Baydin, E. Fidan, K. Aydin, Ö. K. Tuncel, and K. Aslan, "The effect of biomarkers and optic nerve sheath diameter in determining mortality in non-traumatic subarachnoid hemorrhage," Clinical Neurology and Neurosurgery, vol. 207, Article ID 106813, 2021. 
[2] E. Tariq, H. M. Abdullah, U. I. Khan, and M. Omar, "Acute non-traumatic sub-arachnoid hemorrhage in a young female with diabetic ketoacidosis," South Dakota medicine: The Journal of the South Dakota State Medical Association, vol. 74, no. 9, pp. 414-416, 2021.

[3] L. Häni, M. D. Ropelato, F. Wagner et al., "Individualized brain tissue oxygen-monitoring probe placement helps to guide therapy and optimizes outcome in neurocritical care," Neurocritical Care, vol. 35, no. 1, pp. 197-209, 2021.

[4] S. A. Zanelli, M. Abubakar, R. Andris, K. Patwardhan, K. D. Fairchild, and Z. A. Vesoulis, "Early vital sign differences in very low birth weight infants with severe intraventricular hemorrhage," American Journal of Perinatology, 2021.

[5] A. M. Gusdon, C. B. Thompson, K. Quirk et al., "CSF and serum inflammatory response and association with outcomes in spontaneous intracerebral hemorrhage with intraventricular extension: an analysis of the CLEAR-III Trial," Journal of Neuroinflammation, vol. 18, no. 1, p. 179, 2021.

[6] C. Q. Luong, A. D. Nguyen, C. V. Nguyen et al., "Effectiveness of combined external ventricular drainage with intraventricular fibrinolysis for the treatment of intraventricular haemorrhage with acute obstructive hydrocephalus," Cerebrovascular Diseases Extra, vol. 9, no. 2, pp. 77-89, 2019.

[7] Y. Goto, H. Oka, and A. Hino, "Managing intervention for severe intraventricular hemorrhage casting in moyamoya disease: report of two cases," International Journal of Surgery Case Reports, vol. 73, pp. 271-276, 2020.

[8] G.-Q. Wang, S.-Q. Li, Y.-H. Huang et al., "Can minimally invasive puncture and drainage for hypertensive spontaneous Basal Ganglia intracerebral hemorrhage improve patient outcome: a prospective non-randomized comparative study," Military Medical Research, vol. 1, no. 1, p. 10, 2014.

[9] Q.-H. Chen, D. Lin, Q.-G. Yu, and J. Zhou, "Efficacy of lumbar cistern drainage combined with intrathecal antibiotherapy for the treatment of ventriculo-subarachnoid infections following surgery for hypertensive intracerebral hemorrhage," Neurochirurgie, vol. 63, no. 1, pp. 13-16, 2017.

[10] A. Tumturk, Y. Li, Y. Turan, U. Cikla, B. J. Iskandar, and M. K. Baskaya, "Emergency resection of brainstem cavernous malformations," Journal of Neurosurgery, vol. 128, no. 5, pp. 1289-1296, 2018.

[11] B. Y. Choi, O. J. Kim, S. H. Min, J. H. Jeong, S. W. Suh, and T. N. Chung, "Human placenta-derived mesenchymal stem cells reduce mortality and hematoma size in a rat intracerebral hemorrhage model in an acute phase," Stem Cells International, vol. 2018, p. 1658195, Article ID 1658195, 2018.

[12] A. O. Jamshidi, B. Priddy, A. Beer-Furlan, and D. M. Prevedello, "Infradentate approach to the fourth ventricle," Operative Neurosurgery, vol. 16, no. 2, pp. 167-178, 2019.

[13] L. Yang, H. Zhang, X. Wang et al., "Midline suboccipital endoscopic transcerebellomedullary fissure keyhole approach," Journal of Craniofacial Surgery, vol. 28, no. 6, pp. 1603-1606, 2017.

[14] W. Ding, Z. Gu, D. Song, J. Liu, G. Zheng, and C. Tu, "Development and validation of the hypertensive intracerebral hemorrhage prognosis models," Medicine, vol. 97, no. 39, p. e12446, 2018.

[15] J. Ren, X. Wu, J. Huang et al., "Intracranial pressure monitoring-aided management associated with favorable outcomes in patients with hypertension-related spontaneous intracerebral hemorrhage," Translational Stroke Research, vol. 11, no. 6, pp. 1253-1263, 2020.
[16] G. Wang, W. Liu, C. Li, and H. Wang, "Effect of minimally invasive surgery combined with intracranial pressure monitoring on neurological function recovery and quality of life in patients with hypertensive cerebral hemorrhage," American Journal of Tourism Research, vol. 13, no. 7, pp. 8076-8084, 2021.

[17] L. R. Øie, M. A. Madsbu, O. Solheim et al., "Functional outcome and survival following spontaneous intracerebral hemorrhage: a retrospective population-based study," Brain and behavior, vol. 8, no. 10, p. e01113, 2018.

[18] S. P. Klein, D. Bruyninckx, I. Callebaut, and B. Depreitere, "Comparison of intracranial pressure and pressure reactivity index obtained through pressure measurements in the ventricle and in the parenchyma during and outside cerebrospinal fluid drainage episodes in a manipulation-free patient setting," Acta Neurochirurgica Supplement, vol. 126, pp. 287-290, 2018.

[19] A. P. Amar, "Controversies in the neurosurgical management of cerebellar hemorrhage and infarction," Neurosurgical Focus, vol. 32, no. 4, p. E1, 2012.

[20] Z. Y. Xing, H. Y. Zhang, L. G. Sun, J. Zheng, and L. Wang, "Study on the causes, prevention and treatment of complications after transsphenoidal pituitary tumor resection under neuroendoscope," National Medical Journal of China, vol. 99, no. 48, pp. 3803-3807, 2019.

[21] K. Sugiu, K. Tokunaga, S. Ono, A. Nishida, and I. Date, "Rebleeding from a vertebral artery dissecting aneurysm after endovascular internal trapping: adverse effect of intrathecal urokinase injection or incomplete occlusion? -Case report-," Neurologia Medico-Chirurgica, vol. 49, no. 12, pp. 597-600, 2009.

[22] K. N. Fountas, E. Z. Kapsalaki, D. C. Parish et al., "Intraventricular administration of rt-PA in patients with intraventricular hemorrhage," Southern Medical Journal, vol. 98, no. 8, pp. 767-773, 2005.

[23] H. Mitoma, M. Manto, and A. G. Shaikh, "Mechanisms of ethanol-induced cerebellar ataxia: underpinnings of neuronal death in the cerebellum," International Journal of Environmental Research and Public Health, vol. 18, no. 16, 2021.

[24] H. Abe, K. Miki, H. Kobayashi et al., "Unilateral trans-cerebellomedullary fissure approach for occipital artery to posterior inferior cerebellar artery bypass during aneurysmal surgery," Neurologia Medico-Chirurgica, vol. 57, no. 6, pp. 284-291, 2017.

[25] F. Lechanoine, J. F. Zaldivar-Jolissaint, F. Le Lann, and E. De Schlichting, "Endoscopic transfontanellar approach of a cyst anterior to the brainstem crossing the foramen magnumPart I: failure of unique fenestration: 2-dimensional operative video," World Neurosurgery, vol. 146, p. 362, 2021. 